\title{
Highly transparent to truly black electrochromic devices based on an ambipolar system of polyamides and viologen
}

\author{
Huan-Shen Liu', Bo-Cheng Pan ${ }^{1}$, De-Cheng Huang ${ }^{1}$, Yu-Ruei Kung ${ }^{2}$, Chyi-Ming Leu ${ }^{2}$ and Guey-Sheng Liou ${ }^{1}$ \\ A novel electrochromic device (ECD) based on an electroactive ambipolar system was constructed and designed through an \\ absorption-complementary approach. The system consisted of electroactive polyamides (PAs) with $N, N, N^{\prime}, N^{\prime}$-tetraphenyl-p- \\ phenylenediamine (TPPA) and tetraphenylbenzidine (TPB) units in the backbone and heptyl viologen (HV) in the supporting \\ electrolyte. Each of the electrochromic materials (ECMs), including TPPA-PA, TPB-PA and HV, provided one of the three primary \\ colors that merged into a black color. Because of the suitable counter electrode materials used in this study, the overall \\ operating voltage was effectively reduced; thus, the electrochemical stability and lifetime of the ECD were greatly enhanced. \\ Furthermore, the whole system was completely transparent in its neutral or bleaching state, and the transmittance was reduced \\ to only $6 \%$ in the colored state in both the visible and near-infrared (NIR) regions. The ECD demonstrated a high $L^{*}$ change \\ $\left(\Delta L^{*}\right)$ of 81 and a significant transmittance change $(\Delta T)$ of $60 \%$ in the visible region. Thus, through the excellent combination \\ of the electrochromic and ambipolar characteristics of the system, a genuine 'highly transparent to truly black' ECD was \\ successfully fabricated, implying the great potential of this device as a shutter in transparent displays and related devices. \\ NPG Asia Materials (2017) 9, e388; doi:10.1038/am.2017.57; published online 16 June 2017
}

\section{INTRODUCTION}

Display technology has developed for nearly a century. Since the 1920 s, the majority of display materials were based on cathode ray tubes that lasted for several decades until they were replaced by plasma liquid crystal displays and solid-state devices, such as organic lightemitting diodes ${ }^{1,2}$ and light-emitting diodes. With the advancement of technologies and consumer choices, a wide range of display products have been rapidly developed. Now, display trends are moving to the next generation of 'flexible and transparent' ${ }^{3}$ displays.

Transparent displays, as the name indicates, are displays with a high degree of transparency. When they are not displaying, they behave just like glass with high transparency, while they perform with high resolution and contrast in their working states. Such characteristics allow them to have a large variety of applications in electronic devices, such as smart glasses (Google Glass), wearable devices and head-up displays. These applications are expected to have a huge market. Although transparent display technologies have currently already advanced, the typical and fatal obstacle of these technologies is their poor contrast. This problem is caused by light interference from the back of the displays that significantly reduces the vividness of the colors and contrast., ${ }^{4,5}$ To solve this critical issue, shutters that can cooperate with transparent displays are necessary. High optical contrast between the bleaching and coloring states, quick response capability and long-term stability are the essential features for an excellent shutter, and electrochromic devices (ECDs) might be a judicious and representative candidate. $^{6-8}$

Electrochromic materials (ECMs) have advantages of high contrast of transmittance, low driving voltage and bistable characteristics, indicating their great market opportunity in green energy applications and the display industry. However, ECMs with high contrast $\left(\Delta L^{\star}\right)$ in the CIE $L^{*} a^{*} b^{*}$ coordinates have not been easily obtained in the past decade, ${ }^{9-13}$ and the adjective 'black-to-transmissive' was first termed by Reynolds and co-workers in 2008. ${ }^{12}$ They developed readily oxidized conjugated polymers with extensive absorption bandwidths extending over the entire visible spectrum that could be totally bleached in their oxidized states. This system showed a rather high optical contrast ratio, $\Delta L^{*}$ value, of up to $53(19 \rightarrow 72)$.

Recently, most of the ECMs with high contrast have been reported based on conducting polymers, ${ }^{14-21}$ with only a minor amount of studies using small molecules 22,23 or metallo-supramolecular polymers. ${ }^{24}$ Although there are many research groups dedicated to this research area, so far, a $\Delta L^{\star}$ of $>60$ over the whole visible spectrum has not been obtained. This goal is especially important for ECDs that are highly transparent and colorless in their neutral states without any applied potential.

${ }^{1}$ Functional Polymeric Materials Laboratory, Institute of Polymer Science and Engineering, National Taiwan University, Taipei, Taiwan and ${ }^{2}$ Material and Chemical Research Laboratories, Industrial Technology Research Institute, Hsinchu, Taiwan

Correspondence: Professor G-S Liou, Functional Polymeric Materials Laboratory, Institute of Polymer Science and Engineering, National Taiwan University, 1 Roosevelt Road, 4th Sec., Taipei 10617, Taiwan.

E-mail: gsliou@ntu.edu.tw

Received 5 February 2017; revised 3 March 2017; accepted 8 March 2017 


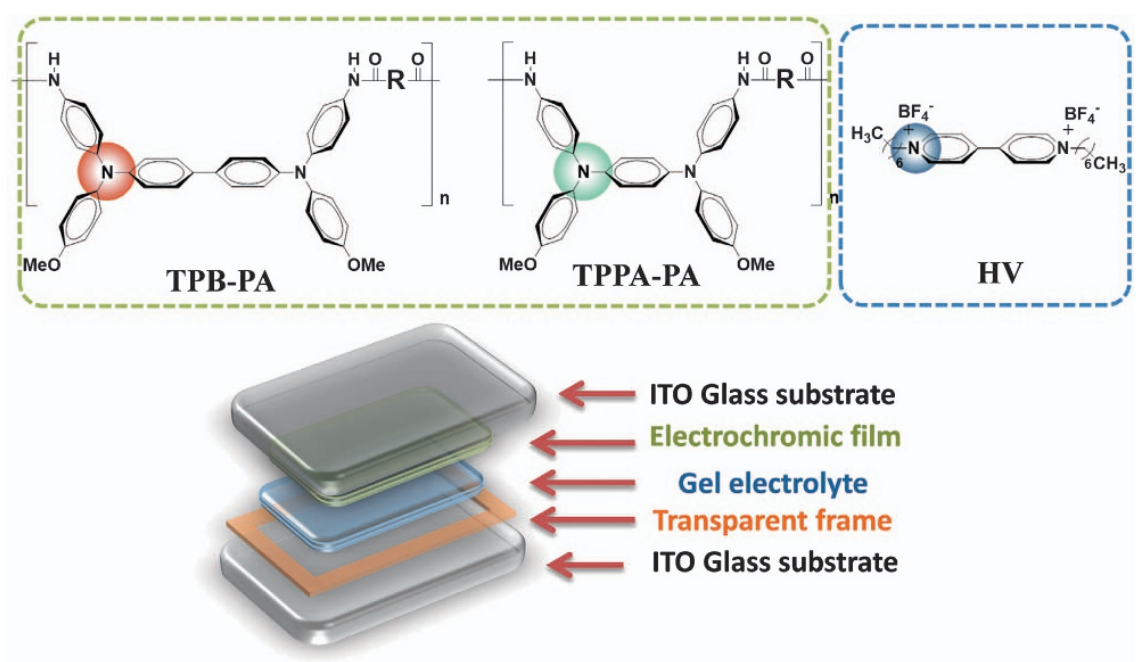

Figure 1 Schematic diagram of the electrochromic device (ECD) based on the ambipolar electrochromic materials (ECMs).
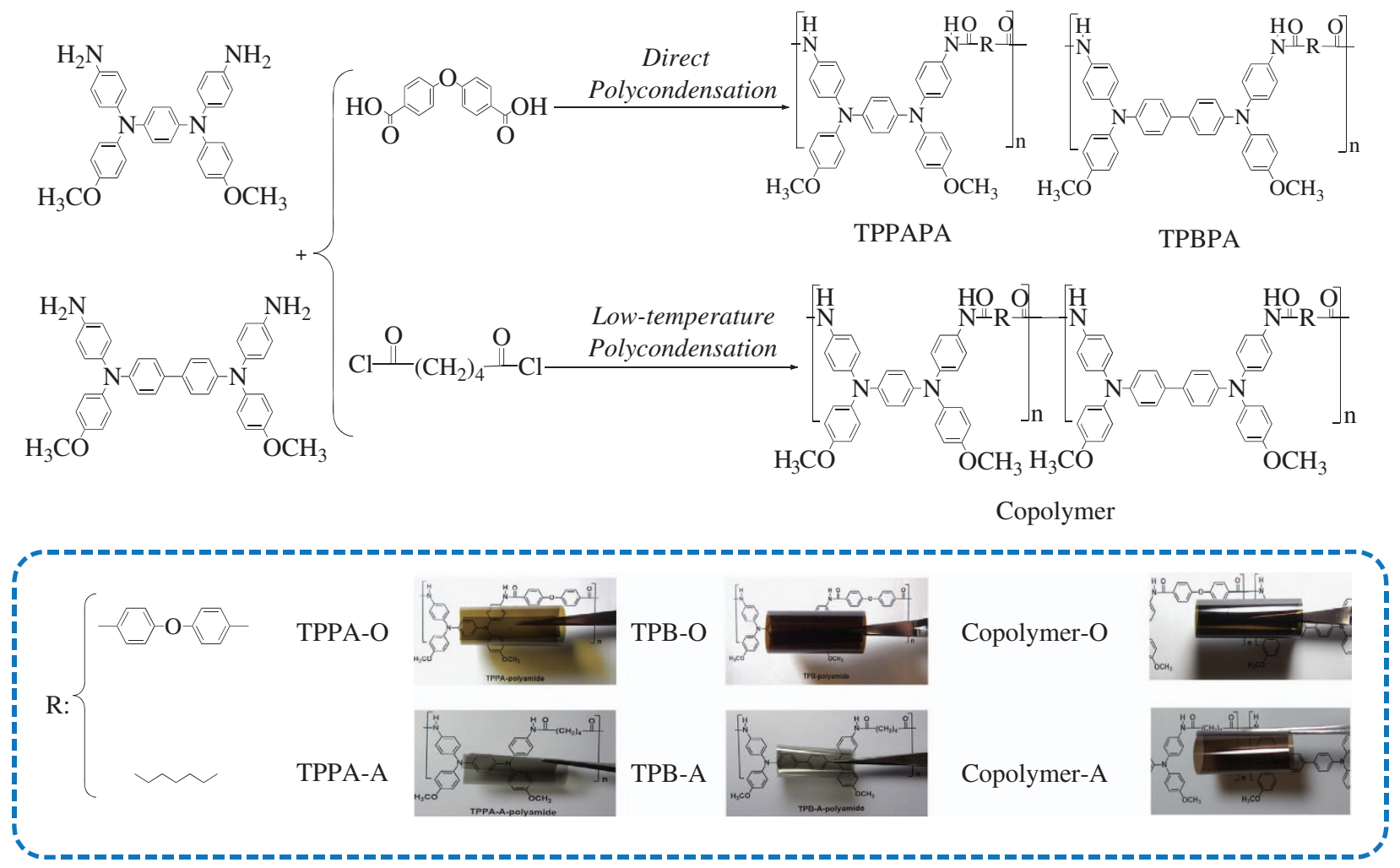

Scheme 1 Synthesis of the polyamides (film thickness: $25 \mu \mathrm{m}$ ).

Therefore, a 'highly transparent to truly black' ECD was elaborately designed and fabricated based on an ambipolar system composed of electroactive polyamides (PAs) derived from $N, N, N^{\prime}, N^{\prime}$-tetraphenyl-pphenylenediamine $(\mathrm{TPPA})^{25}$, tetraphenylbenzidine $(\mathrm{TPB})^{7,26}$ and heptyl viologen (HV) $)^{6,27}$ (Figure 1) to balance the charge, decreasing the driving voltage and enhancing other properties.

By the judicious combination of these three ECMs, the obtained ECDs are not only colorless with high transparency in their neutral states but also produce three primary colors (green, blue and red) by altering their redox states, significantly reducing their degree of transmittance. In addition, according to the CMYK color model, the final color is black after subtracting red, green and blue in the visible spectrum. Therefore, an ECD with a high $\Delta L^{\star}$ of 81 and a significant $\Delta T$ of $>60 \%$ over the whole visible spectrum was achieved. Furthermore, the transmittance could also be reduced to $<5 \%$ in both the near-infrared (NIR) and visible regions in the colored redox states.

\section{EXPERIMENTAL PROCEDURES}

The PAs were synthesized from diamine monomers and dicarboxylic acid (or diacid chloride) via a conventional low-temperature solution polycondensation or direct polycondensation, as shown in Scheme 1. The synthesis of the 


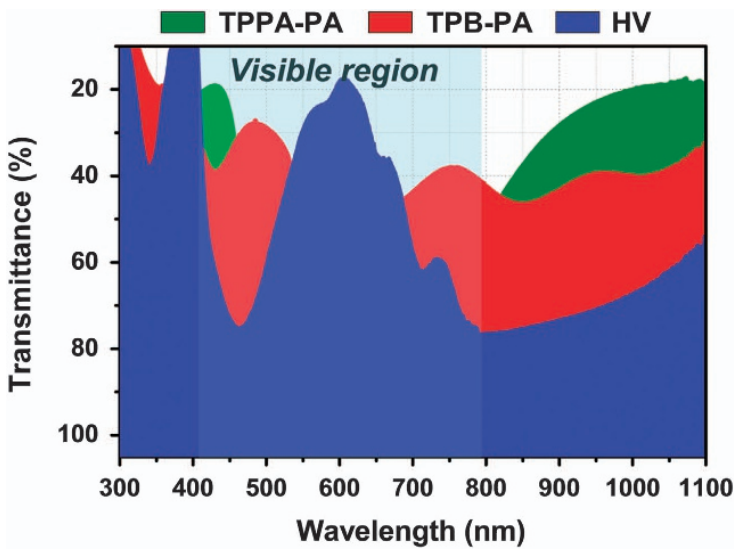

Figure 2 Schematic diagram of the electrochromic device (ECD) based on the ambipolar electrochromic materials (ECMs).

PAs based on a TPPA unit was used to demonstrate the ordinary synthetic method for the following experimental details. The thermal properties, solubility behaviors, molecular weights and inherent viscosities of the resulting PAs are listed in the Supplementary Information.

\section{RESULT AND DISCUSSION}

Basic properties of PAs

A series of PAs was readily prepared via low-temperature solution polycondensation and direct polycondensation from two aromatic diamines and dicarboxylic acid or diacid chloride (as shown in Scheme 1). The molecular weights and solubility behaviors of the obtained polymers are summarized in Supplementary Tables S1 and S2, respectively. These polymers were very soluble in polar aprotic organic solvents owing to the bulky structure of the arylamines along the backbones of the PAs, indicating that these PAs should be easily implemented in solution casting for practical applications. The thermal properties of the PAs were surveyed by thermogravimetric analysis(Supplementary Figure S1) and differential scanning calorimetry (Supplementary Figure S2), and the data are summarized in Supplementary Table S3. All the synthesized PAs displayed good thermal stabilities without significant weight losses up to $400{ }^{\circ} \mathrm{C}$ under air or nitrogen atmospheres even with the aliphatic moieties. The carbonized residues (char yields) of the PAs with aromatic backbones in a nitrogen atmosphere were $>60 \%$ at $800{ }^{\circ} \mathrm{C}$, but the char yields of the corresponding PAs with aliphatic units were only $\sim 30 \%$ at $800^{\circ} \mathrm{C}$. The glass-transition temperatures $\left(T_{\mathrm{g}}\right)$ of the PAs were in the range of $170-230^{\circ} \mathrm{C}$, indicating the rigidities of the polymer chains.

\section{Electrochemical properties of the ECMs}

The electrochemical properties of the anodic electrochromic PAs were surveyed by cyclic voltammetry $(\mathrm{CV})$ that was conducted by casting a film on an indium tin oxide-coated glass slide $(12 \mathrm{~mm} \times 5 \mathrm{~mm})$ as a working electrode in propylene carbonate and using $0.1 \mathrm{M} \mathrm{LiBF}_{4}$ as a supporting electrolyte under a nitrogen atmosphere. The CV diagrams for the PAs are depicted in Supplementary Figure S3a. These PAs exhibited two reversible oxidation peaks, indicating that electrons could be removed in an ordered manner from the two redox centers bridging the phenylene or biphenylene units; and similar potentials ranging from 0.40 to $0.70 \mathrm{~V}$ were needed for the first oxidation step to transform $\mathrm{PA}^{0}$ into $\mathrm{PA}^{1 .+}$. In addition, the redox behavior of the cathodic colored $\mathrm{HV}$ was also measured by $\mathrm{CV}$, as shown in Supplementary Figure S3b, revealing a reversible reduction step at a potential of $\sim-0.50 \mathrm{~V}$. According to the $\mathrm{CV}$ results, the first redox potentials of the anodic (PA) and cathodic (HV) ECMs did not match well with each other, but the results of the ECDs might be different because of a different redox environment, such as electrolyte and solvent, and this will be further investigated for the ECDs.

\section{Electrochemical properties of the ECDs}

Because of the similar electrochemical behaviors of the corresponding PAs derived from the aromatic and aliphatic dicarboxylic acids, the ECDs based on the aromatic PAs were used as examples to illustrate the general CV results. The electrochemical behaviors of TPPA-O and TPB-O ECDs were investigated by CV, as shown in Supplementary Figure S4. The first oxidation potentials from the neutral forms to the cationic radicals for the ECDs increased (TPPA-O ${ }^{1 .+}: 0.50$ to $1.50 \mathrm{~V}$ and TPB-O ${ }^{1 .+}: 0.70$ to $1.70 \mathrm{~V}$ ) because of the gel electrolyte and larger size of the electrode $(25 \mathrm{~mm} \times 20 \mathrm{~mm})$. The rate of ion diffusion decreased in the gel electrolyte system, resulting in a reduction of the charge-exchange rate that leads to the requirement of higher voltages to drive the entire devices. Thus, the power consumption of the ECDs increased and the response capability decreased.

To improve the drawbacks created by the ECDs with the gel electrolyte, the cathodic electrochromic material HV was introduced into the gel electrolyte layer of the ECDs. The representative CV diagrams of TPPA-O/HV and TPB-O/HV ECDs are depicted in Supplementary Figures S5a and b, demonstrating reversible redox steps with much lower oxidative potentials (TPPA-O ${ }^{1 .+} / \mathrm{HV}$ ECD: $0.90 \mathrm{~V}$ and TPB-O ${ }^{1++} / \mathrm{HV}$ ECD: $1.10 \mathrm{~V}$ ) than the corresponding devices without $\mathrm{HV}$. This effect could be ascribed to $\mathrm{HV}^{2+}$ having the ability to accept electrons from PA moieties during the oxidation process, and the resulting $\mathrm{HV}^{1+}$ could also contribute electrons back to $\mathrm{PA}^{1 .+}$ during the reduction process to the original neutral state (Supplementary Figures S5c and d). Thus, adding HV as an efficient charge-trapping molecule in the electrolyte layer not only greatly reduced the working voltage but also enhanced the performance of the overall system.

\section{Spectroelectrochemistry}

Spectroelectrochemical measurements were used to assess the optical behaviors of the electrochromic materials. The PA film was cast onto an indium tin oxide-coated glass slide (sheet resistance: $5 \Omega \mathrm{sq}^{-1}$, transmittance: $80 \%$ at $550 \mathrm{~nm}$, as shown in Supplementary Figure S6), and a homemade electrochemical cell was built from a commercial ultraviolet-visible (UV-vis) cuvette. The cell was set in the optical path of the light beam in a UV-vis-NIR spectrophotometer that allowed us to obtain electronic absorption spectra during the electrochemical experiments in a $0.1 \mathrm{M} \mathrm{LiBF} /$ propylene carbonate solution. The optical absorbance spectra of the PA films, HV and ECDs matched to applied potentials are shown in Supplementary Figures S7, respectively. All the electroactive PAs were colorless and transparent at the bleaching state $(0 \mathrm{~V})$. Upon oxidation, the absorbance at a specific wavelength was greatly enhanced and exhibited unique spectral absorption for each PA. The detailed information of the spectroelectrochemical properties for these materials is in the Supplementary Information. In addition, according to the results of Supplementary Figure S9, the working voltage of both the anodic ECMs (PAs) and the cathodic ECM (HV) could be successively matched and demonstrated excellent complementary color-merging effects. When overlaying the absorption spectra of TPPA-PA (peaks at $\sim 430$ and $600 \mathrm{~nm}$ in the visible region that are associated with a green color at the first oxidation step), TPB-PA (peak at $\sim 486 \mathrm{~nm}$, associated with a red color at the first oxidation step) and $\mathrm{HV}$ (broad band centered at $\sim 603 \mathrm{~nm}$, associated with a blue color at the 
a

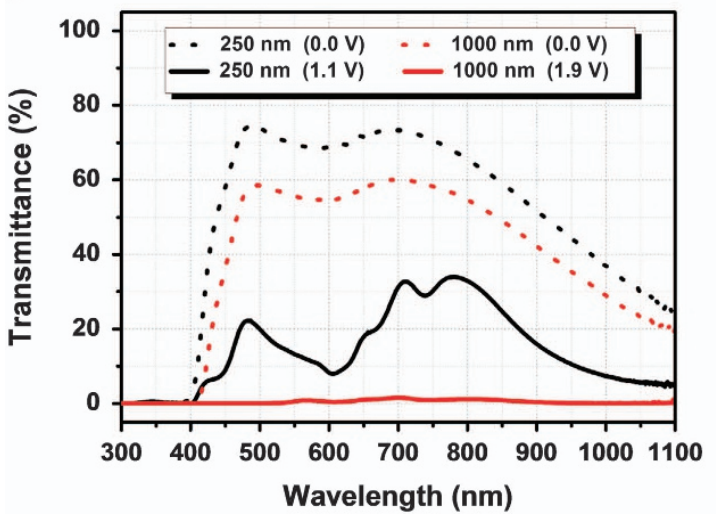

b

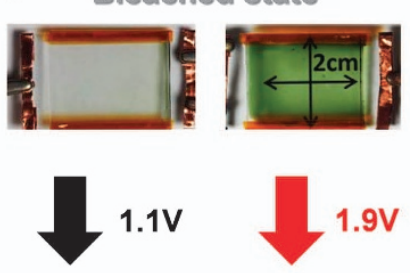

Colored state

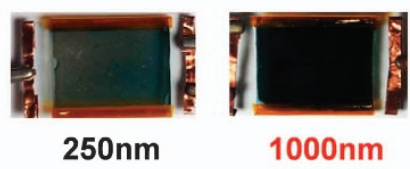

Figure 3 (a) Ultraviolet-visible (UV-vis) spectra of the Blending-O electrochromic devices (ECDs; air as reference) with different thicknesses (250 nm; $1 \mu \mathrm{m}$ ) on indium tin oxide (ITO)-coated glass substrates (coated area: $25 \mathrm{~mm} \times 20 \mathrm{~mm}$; containing $0.5 \mathrm{mg}$ heptyl viologen (HV) as a cathodic electrochromic material $(E C M))$ in propylene carbonate with $3 \mathrm{wt} \% \mathrm{LiBF}_{4}$ as the supporting electrolyte, and (b) photos of the ECDs in their bleached states and colored states.

a

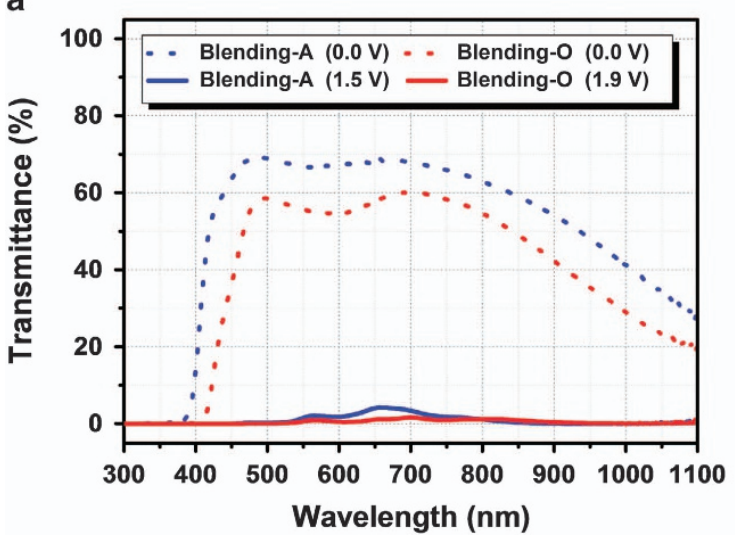

b
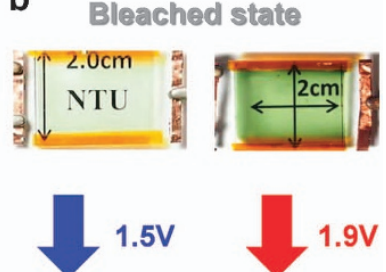

Colored state

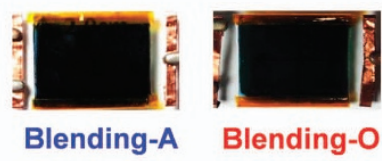

Figure 4 (a) Ultraviolet-visible (UV-vis) spectra of the TPPA-TPB PA blending electrochromic devices (ECDs; air as reference) with different backbone structures (aromatic Blending-O and aliphatic Blending-A) on indium tin oxide (ITO)-coated glass substrates (coated area: $25 \mathrm{~mm} \times 20 \mathrm{~mm}$; thickness: $1 \mu \mathrm{m}$; containing $0.5 \mathrm{mg}$ heptyl viologen (HV) as a cathodic electrochromic material (ECM)) in propylene carbonate with 3 wt\% $\mathrm{LiBF}_{4}$ as the supporting electrolyte, and (b) photos of the ECDs at their bleached states and colored states.

first reduction step), their absorption range almost completely covered the whole visible spectrum, as shown in Figure 2.

\section{Material integration}

To combine the spectroelectrochemical behaviors of TPPA-PA and TPB-PA, copolymerization and blending of the PAs were used, and the CV diagrams of integrated ECDs are described in Supplementary Figure S10. Both the ECDs derived from copolymerization (Copolymer-O) and polymer blending (Blending-O) exhibited similar reversible redox behaviors, and two oxidation potentials for both the Copolymer-O (1.0 and 1.1 V) and Blending-O (1.0 and 1.1 V) ECDs were observed, confirming the successful integration of the TPPA and TPB electrochromic units. UV-vis-NIR transmittance curves correlated to the applied potentials of the Copolymer-O and Blending-O ECDs are summarized in Supplementary Figure S11. The spectroelectrochemical characterizations of all ECMs are included in the UV-vis-NIR spectra, indicating that the ambipolar materials could complement each other and match well at the same redox potential $(1.1 \mathrm{~V})$.

In many previous reports, ${ }^{28-30}$ the stabilities of viologen-based ECDs were poor because of irreversible bleaching processes from the agglomeration of $\mathrm{V}^{1+}$ on the electrodes during operation. Therefore, we chose a longer carbon chain $\mathrm{HV}$ to increase the steric effects and reduce the chance of forming an agglomerate on the electrode. In addition, our ambipolar system ECD could further help solve this problem because the anodic and cathodic ECMs could serve as chargestorage layers to each other to enhance the electrochromic response ability of the ECD. As shown in Supplementary Figure S12a, the reversible electrochromic behavior of $\mathrm{HV}$ was observed by its characteristic absorption peaks $(603 \mathrm{~nm})$ for our Blending-O ECD. The electrochromic coloration efficiency $(\eta=\delta \mathrm{OD} / \mathrm{Q})$ and injected charge (electroactivity) after 100 switching steps are summarized in Supplementary Table S4. The Blending-O ECD was found to exhibit high stability and reserved $>94 \%$ of its electroactivity after switching 100 times between 1.1 and $-1.1 \mathrm{~V}$ (Supplementary Figure S12b). Although the combination of these two approaches can successfully merge the characteristics of the ECMs, a more convenient method for parameter regulation is polymer blending. Thus, the subsequent evaluation will focus on the polymer blending ECDs.

\section{Thickness effects}

The Blending-O ECD (film thickness: $250 \mathrm{~nm}$ ) was colorless and transparent at its neutral state $(0.0 \mathrm{~V})$. After coloration, the transmittance over the whole visible spectrum decreased with a broad absorption band in the NIR and an obvious color change. However, the obtained ECD 

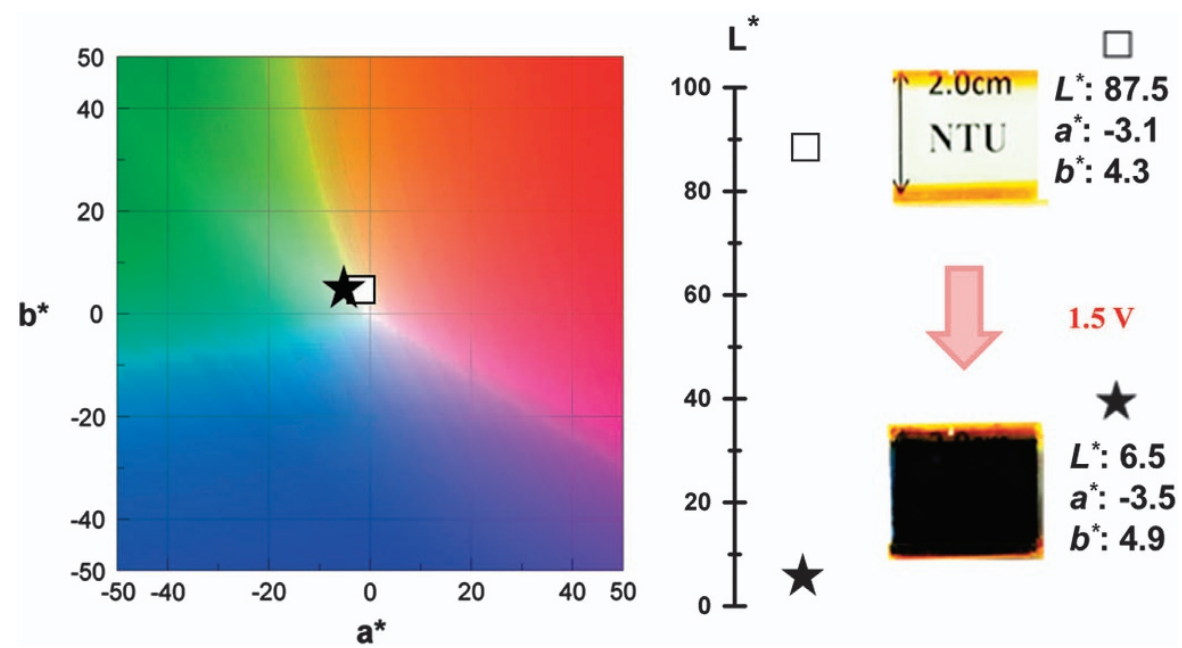

Figure 5 CIE 1976 color diagram of the Blending-A electrochromic device (ECD; coated area: $25 \mathrm{~mm} \times 20 \mathrm{~mm}$; thickness: $1 \mu \mathrm{m}$; containing $0.5 \mathrm{mg}$ heptyl viologen $(\mathrm{HV})$ as a cathodic electrochromic material $(\mathrm{ECM}))$ in propylene carbonate with $3 \mathrm{wt} \% \mathrm{LiBF}_{4}$ as the supporting electrolyte at an applied potential of $1.5 \mathrm{~V}$ for $20 \mathrm{~s}$.
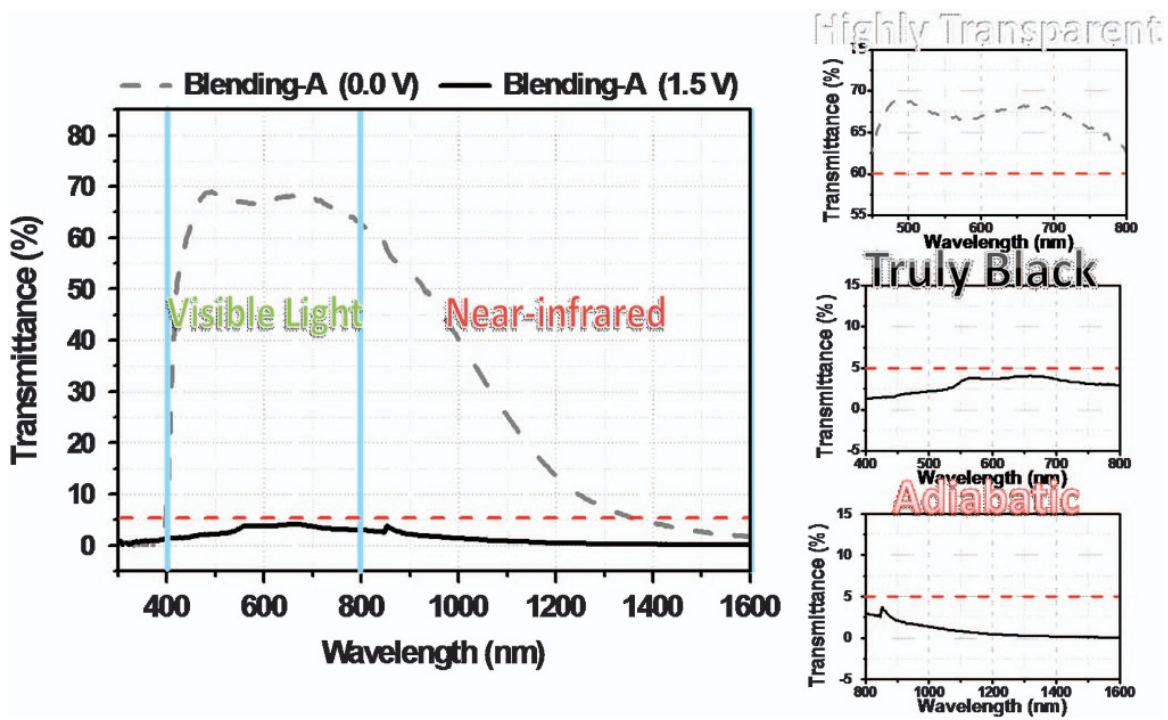

Figure 6 Ultraviolet-visible-near-infrared (UV-vis-NIR) spectra of the Blending-A electrochromic device (ECD; air as reference; coated area: $25 \mathrm{~mm} \times 20 \mathrm{~mm}$; thickness: $1 \mu \mathrm{m}$; containing $0.5 \mathrm{mg}$ heptyl viologen $(\mathrm{HV})$ as a cathodic electrochromic material $(\mathrm{ECM})$ ) in propylene carbonate with 3 wt\% $\mathrm{LiBF}_{4}$ as the supporting electrolyte.

still did not reach an intense black color at this thickness. Thus, the Blending-O ECDs based on different thicknesses of PA films were prepared in order to obtain a higher degree of darkening at the colored state, and the results are summarized in Supplementary Figure S13 to S15. According to the results, the ECD revealed minimal transparency when the thickness of the PA film reached $1 \mu \mathrm{m}$. As depicted in Figure $3 \mathrm{a}$ and Supplementary Figure S13, the transmittance over the visible and NIR regions was effectively reduced to only $1 \%$ during the colored state (by applying a voltage from 0.0 to $1.9 \mathrm{~V}$ ). As shown in the photos in Figure 3b, we observed that the Blending-O ECD (film thickness: $1 \mu \mathrm{m}$ ) turned 'truly black' at the colored state. This excellent performance is attributed to the increased thickness of Blending-O film. Despite this ECD showing excellent visible light absorption at its colored state, the transmittance over the visible spectrum was reduced to only $55 \%$ in the neutral form, indicating that the transparency of the ECD was obviously sacrificed at the bleached state.

\section{Chemical structure effects}

To solve the dilemma of the integrated ECD caused by increasing the thickness of the Blending-O film to $1 \mu \mathrm{m}$, modification of the PA structures was attempted, and the aromatic PAs (TPPA-O and TPB-O) synthesized from aromatic dicarboxylic acid were replaced by partially aromatic PAs (TPPA-A and TPB-A) produced by aliphatic diacid that could effectively reduce the conjugated length and suppress intra- and intermolecular charge transfer, resulting in improved transmittance over the visible spectrum in the neutral state, as shown in Supplementary Figure S16 and Figure 4. The transmittance in the visible region reached up to $65 \%$ (air as reference) for the Blending-A ECD in the bleached state. In addition, the transmittance in the colored state decreased to $<5 \%$ in both the visible and NIR regions. Moreover, the dynamic change of the transmittance curves correlated to the coloring time for the Blending-A ECD, as depicted in Supplementary Figure S17, and an extremely significant transmittance change $(\Delta T)$ higher than $60 \%$ in the 
visible region $(430-800 \mathrm{~nm})$ and a high $L^{*}$ variation $\left(\Delta L^{*}\right)$ of 81 were achieved by the ambipolar Blending-A ECD, as shown in Figure 5.

Furthermore, the response time and long-term stability were also investigated and are summarized in Supplementary Figure S16. The obtained ECD attained up to $90 \%$ of the coloring in $20 \mathrm{~s}$ with a significant $\Delta T$ and exhibited high electrochemical reversibility even over $5 \mathrm{~h}$ in the switched-on state. These results illustrate a novel and facile approach for tuning the transmission regions from highly transparent $(T>65 \%)$ to 'truly black' $(T<5 \%)$, as shown in Figure 6 , and for tuning the electrochromic stability, indicating the high potential for this device for use in transparent displays and related optical devices.

\section{CONCLUSIONS}

A high-performance ECD was successfully fabricated from three different types of electrochromic materials, TPPA-PA, TPB-PA and $\mathrm{HV}$. By introducing $\mathrm{HV}$ as an efficient charge-trapping layer, the working voltage was greatly reduced, and the performance of the overall system was also enhanced. The Blending-A ECD system exhibited a ultra-high contrast from the bleaching state (highly transparent neutral form), with transmittance in the visible region $>65 \%$, to the colored state ('truly black' redox form), with transmittance of only $<5 \%$ in both the visible and NIR regions. High $\Delta L^{*}(81)$ and $\Delta T(60.0 \%$ in the visible region) were achieved by the colorless ECD. Thus, this ambipolar system with a low driving voltage and a high optical contrast ratio in both the visible and NIR regions could be claimed to be a truly 'transmissive-to-black' ECD, implying the great potential of this device as a shutter for transparent displays and energy-saving devices.

\section{CONFLICT OF INTEREST}

The authors declare no conflict of interest.

\section{ACKNOWLEDGEMENTS}

We gratefully acknowledge the Ministry of Science and Technology of Taiwan for financial support.

1 Tseng, F. M., Cheng, A. C. \& Peng, Y. N. Assessing market penetration combining scenario analysis, Delphi, and the technological substitution model: the case of the OLED TV market. Technol. Forecast Soc. 76, 897-909 (2009).

2 Mentley, D. E. State of flat-panel display technology and future trends. Proc. IEEE 90, 453-459 (2002).

3 Markets and Opportunities for Transparent Displays. http://ntechresearch.com/market_reports/markets-and-opportunities-for-transparent-displays-2014-to-2021\#sthash. OnMOpzz5.dpuf LG Display, Transparent Flexible Display http://www.lgdisplay.com/eng/ product/technology/flexibleDisplay.

4 Lin, J. N., Chen, C. H. \& Su, C. W. Transparent display device and displaying method thereof. US 20150355514 A1 (2015).

5 Schaefer, E., Vogt, R., Brankl, M., Zeiner, M., Keller, M., Schubert, W., Warmuth, S., Kloiber, T., Blume, D. \& Herrmann, J. Transparent display device. US 20150338715 A1 (2015).

6 Monk, P. M. S. The effect of ferrocyanide on the performance of heptyl viologen-based electrochromic display devices. J. Electroanal. Chem. 432, 175-179 (1997).

7 Platt, J. R. Electrochromism, a possible change of color producible in dyes by an electric field. J. Chem. Phys. 34, 862-863 (1961).

8 Rosseinsky, D. R. \& Mortimer, R. J. Electrochromic systems and the prospects for devices. Adv. Mater. 13, 783-793 (2001).

9 Dyer, A. L., Thompson, E. J. \& Reynolds, J. R. Completing the color palette with sprayprocessable polymer electrochromics. ACS Appl. Mater. Interfaces 3, 1787-1795 (2011).

10 iccli, M., Pamuk, M., Algı, F., Önal, A. M. \& Cihaner, A. A new soluble neutral state black electrochromic copolymer via a donor-acceptor approach. Organic Electronics 11, 1255-1260 (2010).

11 Unur, E., Beaujuge, P. M., Ellinger, S., Jung, J. H. \& Reynolds, J. R. Black to transmissive switching in a pseudo three-electrode electrochromic device. Chem. Mater. 21, 5145-5153 (2009).
12 Beaujuge, P. M., Ellinger, S. \& Reynolds, J. R. The donor-acceptor approach allows a black-to-transmissive switching polymeric electrochrome. Nat. Mater. 7, 795-799 (2008).

13 Yen, H. J., Lin, K. Y. \& Liou, G. S. Transmissive to black electrochromic aramids with high near-infrared and multicolor electrochromism based on electroactive tetraphenylbenzidine units. J. Mater. Chem. 21, 6230-6237 (2011).

14 Chen, C., Hong, X., Xu, T., Lu, L. \& Gao, Y. Electrosynthesis and electrochemical and electrochromic properties of poly(aniline-co-N-methylthionine). J. Electrochem. Soc. 162, G54-G62 (2015).

15 Jarosz, T., Brzeczek, A., Walczak, K., Lapkowski, M. \& Domagala, W. Multielectrochromism of redox states of thin electropolymerised films of poly (3-dodecylpyrrole) involving a black coloured state. Electrochim. Acta. 137, 595-601 (2014)

16 Alamer, F. A., Otley, M. T., Ding, Y. \& Sotzing, G. A. Solid-state high-throughput screening for color tuning of electrochromic polymers. Adv. Mater. 25, 6256-6260 (2013).

17 Beaujuge, P. M., Vasilyeva, S. V., Liu, D. Y., Ellinger, S., McCarley, T. D. \& Reynolds, J. R. Structure-performance correlations in spray-processable green dioxythiophenebenzothiadiazole donor-acceptor polymer electrochromes. Chem. Mater. 24, 255-268 (2012).

18 Wang, Y., Wei, H., Wang, J., Liu, J., Guo, J., Zhang, X., Weeks, B. L., Shen, T. D., Wei, S. \& Guo, Z. Electropolymerized polyaniline/manganese iron oxide hybrids with an enhanced color switching response and electrochemical energy storage. J. Mater. Chem. A 3, 20778-20790 (2015).

19 Wei, H., Zhu, J., Wu, S., Wei, S. \& Guo, Z. Electrochromic polyaniline/graphite oxide nanocomposites with endured electrochemical energy storage. Polymer 54, 1820-1831 (2013)

20 Wei, H., Yan, X., Li, Y., Gu, H., Wu, S., Ding, K., Wei, S. \& Guo, Z. Electrochromic poly (DNTD)/WO3 nanocomposite films via electorpolymerization. J. Phys. Chem. C 116, 16286-16293 (2012).

21 Wei, H., Yan, X., Li, Y., Wu, S., Wang, A., Wei, S. \& Guo, Z. Hybrid electrochromic fluorescent poly(DNTD)/CdSe@ZnS composite films. J. Phys. Chem. C 116, 4500-4510 (2012).

22 Ah, C. S., Song, J., Cho, S. M., Kim, T. Y., Kim, H. N., Oh, J. Y., Chu, H. Y. \& Ryu, H. Double-layered black electrochromic device with a single electrode and long-term bistability. Bull. Korean Chem. Soc. 36, 548-552 (2015).

23 Weng, D., Shi, Y., Zheng, J. \& Xu, C. High performance black-totransmissive electrochromic device with panchromatic absorption based on TiO2supported viologen and triphenylamine derivatives. Org. Electron. 34, 139-145 (2016).

24 Hsu, C. Y., Zhang, J., Sato, T., Moriyama, S. \& Higuchi, M. Black-to-transmissive electrochromism with visible-to-near-infrared switching of a Co(II)-based metallosupramolecular polymer for smart window and digital signage applications. ACS Appl. Mater. Interfaces 7, 18266-18272 (2015).

25 Yen, H. J. \& Liou, G. S. Solution-processable novel near-infrared electrochromic aromatic polyamides based on electroactive tetraphenyl-p-phenylenediamine moieties. Chem. Mater. 21, 4062-4070 (2009).

26 Yen, H. J. \& Liou, G. S. Solution-processable triarylamine-based electroactive high performance polymers for anodically electrochromic applications. Polym. Chem. 3, 255-264 (2012).

$27 \mathrm{Wu}$, J. H. \& Liou, G. S. High-performance electrofluorochromic devices based on electrochromism and photoluminescence-active novel poly(4-cyanotriphenylamine). Adv. Funct. Mater. 24, 6422-6429 (2014).

28 Lu, H. C., Kao, S. Y., Chang, T. H., Kung, C. W. \& Ho, K. C. An electrochromic device based on Prussian blue, self-immobilized vinyl benzyl viologen, and ferrocene. Sol. Energy Mater. 147, 75-84 (2016).

29 Bewick, A., Lowe, A. C. \& Wederell, C. W. Recrystallisation process in viologen-based electrochromic deposits: voltammetry coupled with rapid time-resolved spectroscopy. Electrochim. Acta 28, 1899-1902 (1983).

30 Hoshino, K., Nakajima, R. \& Okuma, M. Improved electrochromic performance of viologen at an ITO-nanoparticle film electrode. Appl. Surf. Sci. 313, 569-576 (2014).

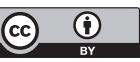

This work is licensed under a Creative Commons Attribution 4.0 International License. The images or other third party material in this article are included in the article's Creative Commons license, unless indicated otherwise in the credit line; if the material is not included under the Creative Commons license, users will need to obtain permission from the license holder to reproduce the material. To view a copy of this license, visit http:// creativecommons.org/licenses/by/4.0/

(C) The Author(s) 2017

Supplementary Information accompanies the paper on the NPG Asia Materials website (http://www.nature.com/am) 